\title{
DESIGN, SYNTHESIS, CHARACTERIZATION, AND CYTOTOXICITY EVALUATION OF NEW 4-BENZYL-1,3-OXAZOLE DERIVATIVES BEARING 4-(4-CHLOROPHENYLSULFONYL)PHENYL MOIETY
}

\author{
THEODORA-VENERA APOSTOL ${ }^{1} *$, LAURA-ILEANA SOCEA ${ }^{1}$, CONSTANTIN DRĂGHICI $^{2}$, \\ OCTAVIAN TUDOREL OLARU ${ }^{3}$, GABRIEL ȘARAMET ${ }^{4}$, CRISTIAN ENACHE-PREOTEASA ${ }^{5}$, \\ ŞTEFANIA-FELICIA BĂRBUCEANU ${ }^{1}$
}

\author{
1 “Carol Davila” University of Medicine and Pharmacy, Faculty of Pharmacy, Organic Chemistry Department, 6 Traian \\ Vuia Street, 020956, Bucharest, Romania \\ 2 “Costin D. Nenițescu” Institute of Organic Chemistry, Romanian Academy, 202B Splaiul Independenței Street, 060023, \\ Bucharest, Romania \\ 3 "Carol Davila” University of Medicine and Pharmacy, Faculty of Pharmacy, Pharmaceutical Botany and Cell Biology \\ Department, 6 Traian Vuia Street, 020956, Bucharest, Romania \\ 4 "Carol Davila" University of Medicine and Pharmacy, Faculty of Pharmacy, Pharmaceutical Technology and \\ Biopharmacy Department, 6 Traian Vuia Street, 020956, Bucharest, Romania \\ ${ }^{5}$ National Phytosanitary Laboratory, 11 Voluntari Boulevard, 077190, Voluntari, Ilfov, Romania
}

*corresponding author: theodora.apostol@umfcd.ro

Manuscript received: September 2020

\begin{abstract}
This paper presents the design, synthesis, characterization, and cytotoxicity evaluation of eight organic compounds that have in their molecules the 4-(4-chlorophenylsulfonyl)phenyl moiety: four acyclic precursors derived from phenylalanine (one $\mathrm{N}$ acyl- $\alpha$-amino acid, one $N$-acyl- $\alpha$-amino acyl chloride, and two $N$-acyl- $\alpha$-amino ketones), and four cyclization products: one 1,3-oxazol-5(4H)-one, and three 1,3-oxazoles substituted in 5-position with phenyl, $p$-tolyl, and $m$-xylyl group, respectively. Structures of synthesized compounds were confirmed by elemental analysis and spectral methods (UV-Vis, FT-IR, MS, ${ }^{1} \mathrm{H}-$ and ${ }^{13} \mathrm{C}$-NMR). The compounds purity determination was performed by RP-HPLC. Cytotoxic effect of synthesized compounds was evaluated on Daphnia magna crustacean.
\end{abstract}

\section{Rezumat}

Lucrarea prezintă proiectarea, sinteza, caracterizarea și evaluarea citotoxicității a opt compuși organici care au în moleculele lor fragmentul 4-(4-clorofenilsulfonil)fenil: patru precursori aciclici derivați de la fenilalanină (un $N$-acil- $\alpha$-aminoacid, o clorură de $N$-acil- $\alpha$-aminoacil și două $N$-acil- $\alpha$-aminocetone) și patru produşi de ciclizare: o 1,3-oxazol-5(4H)-onă și trei 1,3oxazoli substituiți în poziția 5 cu grupa fenil, $p$-tolil și respectiv, $m$-xilil. Structurile compușilor sintetizați au fost confirmate prin analiză elementală și metode spectrale (UV-Viz, FT-IR, SM, ${ }^{1} \mathrm{H}-$ și ${ }^{13} \mathrm{C}-\mathrm{RMN}$ ). Determinarea purităţii compuşilor a fost realizată prin RP-HPLC. Efectul citotoxic al compușilor sintetizați a fost evaluat pe crustaceul Daphnia magna.

Keywords: $N$-acyl- $\alpha$-amino acid, 1,3-oxazol-5(4H)-one, 1,3-oxazole, cytotoxicity

\section{Introduction}

Five-membered heterocycles with 1,3-oxazole ring are valuable compounds both for synthetic organic chemistry and medicinal/pharmaceutical chemistry because of the extensive spectrum of biological properties that they present. Numerous representatives of this class are bioactive molecules with analgesic, antiinflammatory [1], antimicrobial [2], antituberculosis [3], antidiabetic [4], anticancer effect [5] etc. Moreover, in nature are present various 1,3-oxazole-based derivatives, which were identified to have significant therapeutic properties, such as analgesic, antiviral, antioxidant, antifungal [6], antibacterial [7], anticancer [8], antimycobacterial action [9] etc. Saturated 1,3oxazol-5(4H)-ones - which are stable, keto form of corresponding 5-hydroxy substituted 1,3-oxazoles have cytotoxic [10], antiviral [11, 12], plant growth regulating activity [13].

Also, acyclic intermediates from $N$-acyl- $\alpha$-amino acids class are known to present mucolytic [14], antihypertensive [15], anticancer [16], antianemic [17], antiulcer [18], vasoconstrictor action [19]. In addition, some $N$-acylated $\alpha$-amino acids are specific antidotes in acute intoxications with antifolates [20], paracetamol [21] etc. Open-chain precursors from $N$-acyl- $\alpha$-amino ketones class display antiviral [22], anti-inflammatory [23] and antithrombotic effect [24].

Besides, the literature study revealed that many diaryl sulfones have several biological properties [25-30]. For example, the structural prototype of this class: Dapsone exhibits remarkable efficient pharmacological 
FARMACIA, 2021, Vol. 69, 2

activity against Mycobacterium leprae. It is also used as part of multidrug therapy in the treatment of patients with all types of leprosy, dermatitis herpetiformis, and other dermatoses, for prophylaxis of Pneumocystis jiroveci pneumonia in immunodeficient patients and prevention of malaria. Notable, compounds resulting from incorporation into the same structure of a diaryl sulfone pharmacophore and of various types of heterocyclic analogues have shown potential biological activities [10, 13, 31, 32].

Taking into consideration these literature data and as a continuation of our research to obtain heterocycles with a 1,3-oxazole ring containing in 2-position a substituent derived from a diaryl sulfone [10, 13, 33], in this study, we present the design, synthesis, and characterization of new organic compounds from $N$ acyl- $\alpha$-amino acids, 1,3-oxazol-5(4H)-ones, $N$-acyl$\alpha$-amino acyl chlorides, $N$-acyl- $\alpha$-amino ketones, and 1,3-oxazoles classes which incorporate a 4-(4-chlorophenylsulfonyl)phenyl moiety into their molecules with the purpose of discovering new active substances and to study the influence of structural changes on biological activity. In this order, newly derivatives were assessed for cytotoxic activity using Daphnia magna bioassay. This screening test is reproducible, fast, simple, cost-effective and can predict the biological action [10].

\section{Materials and Methods}

\section{Chemistry}

General: Melting points (uncorrected) were determined with a Boetius apparatus. UV-Vis spectra were registered for solutions in methanol (in a concentration of about $2.5 \times 10^{-5} \mathrm{M}$ ) on an Analytik Jena AG Specord 40 spectrophotometer. FT-IR spectra were recorded on a Bruker Vertex 70 spectrometer (in $\mathrm{KBr}$ pellets). IR absorption bands intensity is given as: vs = very strong, $\mathrm{s}=$ strong, $\mathrm{m}=$ medium and $\mathrm{w}=$ weak. $\mathrm{NMR}$ spectra were registered on a Varian Gemini 300BB spectrometer at $300 \mathrm{MHz}$ for ${ }^{1} \mathrm{H}-\mathrm{NMR}$ and $75 \mathrm{MHz}$ for ${ }^{13} \mathrm{C}-\mathrm{NMR}$ in DMSO- $\mathrm{d}_{6}$ or $\mathrm{CDCl}_{3}$ as solvents. Chemical shifts $(\delta)$ are reported in ppm downfield of tetramethylsilane used as an internal standard. Coupling constants $(J)$ are expressed in Hz. Abbreviations for multiplicities and descriptors of signals in ${ }^{1} \mathrm{H}-\mathrm{NMR}$ spectra are as follows: $\mathrm{s}=$ singlet, $\mathrm{d}=$ doublet, $\mathrm{dd}=$ doublet of doublets, $\mathrm{t}=$ triplet, $\mathrm{m}=$ multiplet; $\mathrm{b}=$ broad signal. LC-ESI-MS/MS spectra were recorded on a Varian 1200 LC-MS/MS high-performance liquid chromatograph coupled with a triple quadrupole mass spectrometer with an electrospray interface (ESI), by positive and/or negative ionization. Fragments were obtained by collision with argon at different energies up to $50 \mathrm{eV}$. GC-EI-MS analysis was performed using a Fisons Instruments GC 8000 with an electron impact quadrupole, MD 800 mass spectrometer detector. A fused-silica capillary column coated with poly $(5 \%$ diphenyl/95\% dimethylsiloxane) (SLB-5ms, L $\times$ I.D. $30 \mathrm{~m} \times 0.32 \mathrm{~mm}, \mathrm{~d}_{\mathrm{f}} 0.25 \mu \mathrm{m}$ ) was used. A $1 \mu \mathrm{L}$ sample solution in dichloromethane was injected. The flow rate of the carrier gas (helium) was $2 \mathrm{~mL} / \mathrm{min}$. RPHPLC chromatograms were registered on a Beckman System Gold 126 liquid chromatograph, with a System Gold 166 UV-Vis detector, a non-polar chromatography column (type LiChrosorb RP-18, $5 \mu$ m particle size, L $\times$ I.D. $25 \mathrm{~cm} \times 4 \mathrm{~mm}$ ) and a Rheodyne injection system. The flow rate of the mobile phase (a mixture of methanol-water in various proportions) was 1 $\mathrm{mL} / \mathrm{min}$. the purity and retention time $\left(t_{\mathrm{R}}\right)$ of the analysed compounds in minutes (min) are reported. Elemental analysis was performed using a Costech ECS 4010 analyser.

Synthesis and characterization of compounds

Successive reactions used to obtain title compounds are shown in Figure 1. Known raw material, acyl chloride $\mathbf{2}$, was prepared by reacting corresponding carboxylic acid $\mathbf{1}$ with thionyl dichloride. Steiger $\mathrm{N}$-acylation of phenylalanine with acyl chloride $\mathbf{2}$ afforded compound 3. Further, $N$-acyl- $\alpha$-amino acid 3 was cyclodehydrated in presence of ethyl chloroformate and 4-methylmorpholine or of acetic anhydride to saturated 2,4-disubstituted 1,3-oxazol-5(4H)-one 4. Also, $N$-acylated phenylalanine $\mathbf{3}$ was refluxed in thionyl dichloride to acyl chloride 5. Friedel-Crafts reaction of aromatic hydrocarbons (benzene, toluene, $m$-xylene) with saturated azlactone 4 , in presence of aluminium chloride, produced $N$-acyl- $\alpha$-amino ketones 6. Also, the reaction of $\mathrm{N}$-acyl phenylalanyl chloride 5 with benzene was carried out. Subsequently, acyclic intermediates 6 were converted by Robinson-Gabriel synthesis into 2,4,5-trisubstituted 1,3-oxazoles 7 using phosphoryl trichloride or concentrated sulfuric acid in acetic anhydride as cyclodehydrating agents. Depicted chemical structures of novel compounds 3-7 were corroborated by spectral (UV-Vis, FT-IR, MS, ${ }^{1} \mathrm{H}$ and ${ }^{13} \mathrm{C}-\mathrm{NMR}$ ) and elemental analysis data. 


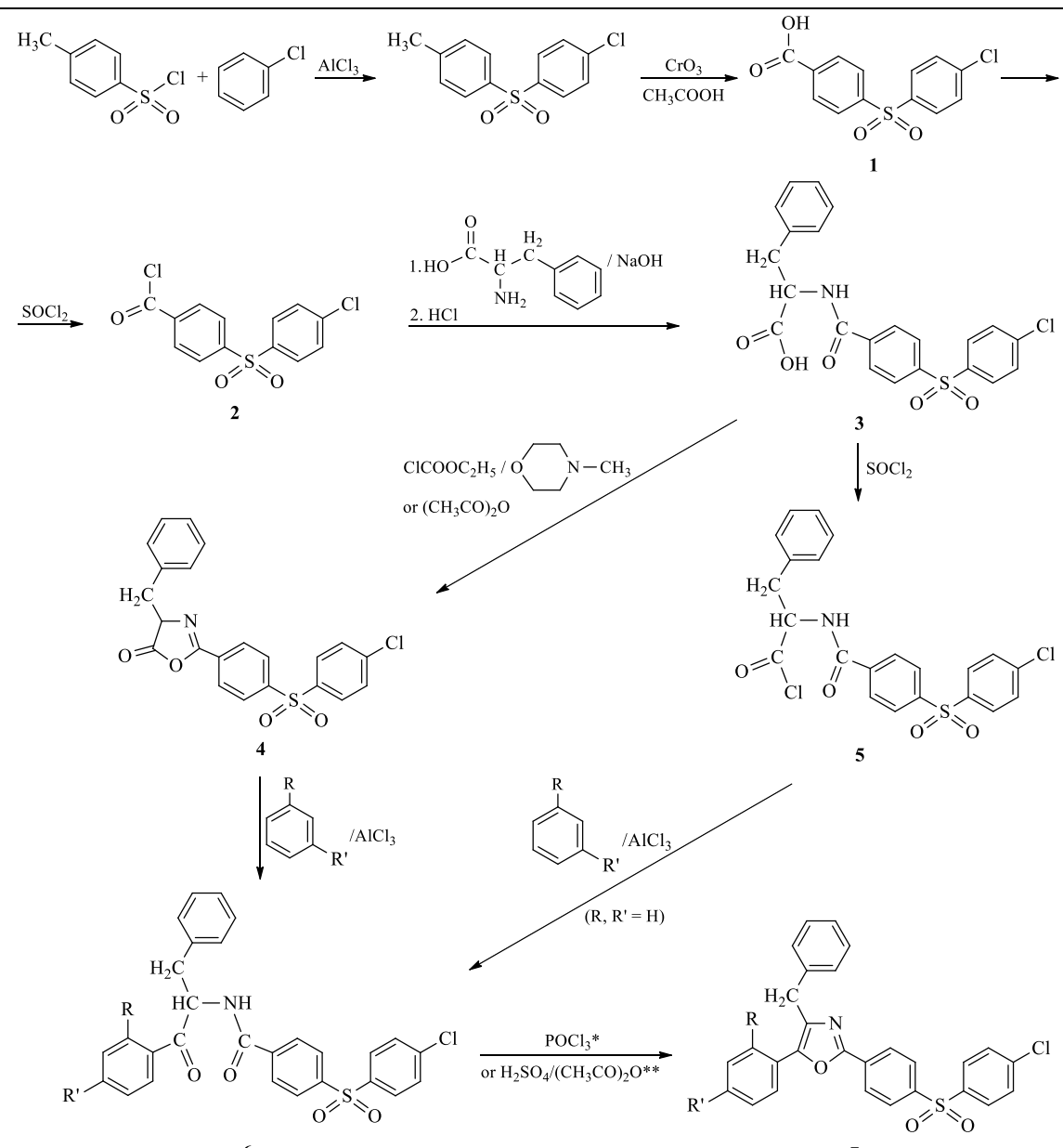

6a-c

6a, 7a: $\mathrm{R}=\mathrm{H}, \mathrm{R}^{\prime}=\mathrm{H}$

6b, 7b: $\mathrm{R}=\mathrm{H}, \mathrm{R}^{\prime}=\mathrm{CH}_{3}$

6c, 7c: $\mathrm{R}, \mathrm{R}^{\prime}=\mathrm{CH}_{3}$

* used for obtaining $\mathbf{7 a - c}$

** used for obtaining $\mathbf{7 b}$

Figure 1.

Multistep reaction sequence for compounds synthesis

Synthesis of 2-[4-(4-chlorophenylsulfonyl)benzamido]3-phenylpropanoic acid $\mathbf{3}$

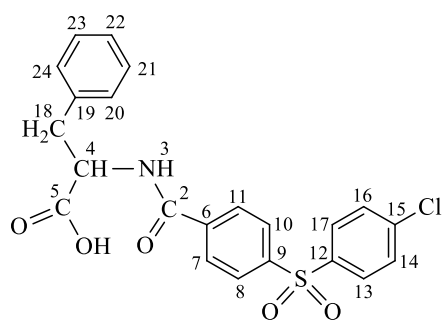

Figure 2.

Compound $\mathbf{3}$ structure with atomic numbering (for NMR assignments)

Phenylalanine (3.30 g, $20 \mathrm{mmol}$ ) was dissolved in $1 \mathrm{~N}$ sodium hydroxide solution $(20 \mathrm{~mL})$. To this solution cooled in an ice bath, a solution of crude 4-(4chlorophenylsulfonyl)benzoyl chloride $2(6.30 \mathrm{~g}, 20$ $\mathrm{mmol})$ in anhydrous dichloromethane $(45 \mathrm{~mL})$ and a
$2 \mathrm{~N}$ sodium hydroxide solution $(10 \mathrm{~mL})$, respectively were added simultaneously, gradually, with agitation, for $30 \mathrm{~min}$. The reaction mixture stirring was continued for $1 \mathrm{~h}$ at room temperature. Then, the aqueous layer was separated and acidified with $2 \mathrm{~N}$ hydrochloric acid. The precipitated solid was separated by filtration, washed with water, dried and purified by recrystallization from water as white acicular crystals; yield $=94 \%$ $(8.35 \mathrm{~g})$; m.p. $=165-166^{\circ} \mathrm{C}$.

UV-Vis $\left(\mathrm{CH}_{3} \mathrm{OH}, \lambda \mathrm{nm}\right)$ (lg $\left.\varepsilon\right): 202.6$ (4.48); 250.2 (4.16).

FT-IR (KBr, $v \mathrm{~cm}^{-1}$ ): 3355s; 3086m; 3066m; 3028m; 2977m; 2937m; 2869m; 2704m; 2639m; 2592m; 2532m; $1734 \mathrm{vs} ; 1623 \mathrm{vs} ; 1567 \mathrm{~s} ; 1548 \mathrm{vs} ; 1494 \mathrm{~m} ; 1478 \mathrm{~m} ; 1447 \mathrm{~m}$; 1324vs; 1309s; 1295s; 1162vs; 852m; 758s.

${ }^{1} \mathrm{H}-\mathrm{NMR}$ (DMSO-d 6 , $\delta$ ppm, $J$ Hz): 3.04 (dd, 14.0, 10.7, 1H, H-18); 3.21 (dd, 14.0, 4.7, 1H, H-18); 4.64 (m, 1H, H-4); 7.16 (bt, 6.6, 1H, H-22); 7.20-7.38 (m, 4H, H-20, H-21, H-23, H-24); 7.70 (d, 8.8, 2H, H-14, H-16); 7.97 (d, 8.5, 2H, H-8, H-10); 7.99 (d, 8.8, 2H, 
FARMACIA, 2021, Vol. 69, 2

H-13, H-17); 8.07 (d, 8.5, 2H, H-7, H-11); 9.00 (d, $8.0,1 \mathrm{H}, \mathrm{NH})$.

${ }^{13} \mathrm{C}-\mathrm{NMR}$ (DMSO-d 6 , $\delta$ ppm): 36.34 (C-18); 54.38 (C-4); 126.48 (C-22); 127.71 (C-8, C-10); 128.29 (C-20, C-24); 128.81 (C-7, C-11); 129.10 (C-21, C23); 129.56 (C-13, C-17); 130.09 (C-14, C-16); 138.04 (C-19); 138.78 (C-6); 139.22 (C-15); 139.55 (C-12); 142.94 (C-9); 165.12 (C-2); 172.86 (C-5).

+ ESI-MS/MS ( $\mathrm{m} / z$, rel. abund. \%): $444\left({ }^{35} \mathrm{Cl}\right) / 446\left({ }^{37} \mathrm{Cl}\right)$ $[\mathrm{M}+\mathrm{H}]^{+} ; 426 / 428(8.3 / 11.3)\left[\mathrm{M}+\mathrm{H}_{-} \mathrm{H}_{2} \mathrm{O}\right]^{+} ; 398 / 400$ (100, BP) [M+H-H $\left.{ }_{2} \mathrm{O}-\mathrm{CO}\right]^{+} ; 279 / 281$ (16.7/23.3) $\left[{ }^{35} \mathrm{ClC}_{6} \mathrm{H}_{4} \mathrm{SO}_{2} \mathrm{C}_{6} \mathrm{H}_{4} \mathrm{CO}\right]^{+} /\left[{ }^{37} \mathrm{ClC}_{6} \mathrm{H}_{4} \mathrm{SO}_{2} \mathrm{C}_{6} \mathrm{H}_{4} \mathrm{CO}\right]^{+}$. -ESI-MS/MS ( $\mathrm{m} / \mathrm{z}$, rel. abund. \%): $442\left({ }^{35} \mathrm{Cl}\right) / 444\left({ }^{37} \mathrm{Cl}\right)$ [M-H] ; 398/400 (76.7/80.6) [M-H-CO $\left.{ }_{2}\right]^{-} ; 307 / 309$ (12.6/11.7) $\quad\left[{ }^{35} \mathrm{ClC}_{6} \mathrm{H}_{4} \mathrm{SO}_{2} \mathrm{C}_{6} \mathrm{H}_{4} \mathrm{CONHCH} \cdot\right]^{-/}$ $\left[{ }^{37} \mathrm{ClC}_{6} \mathrm{H}_{4} \mathrm{SO}_{2} \mathrm{C}_{6} \mathrm{H}_{4} \mathrm{CONHCH} \cdot\right]^{-} ; 294 / 296$ (12.9/11.0) $\left[{ }^{35} \mathrm{ClC}_{6} \mathrm{H}_{4} \mathrm{SO}_{2} \mathrm{C}_{6} \mathrm{H}_{4} \mathrm{CONH}\right] /\left[{ }^{37} \mathrm{ClC}_{6} \mathrm{H}_{4} \mathrm{SO}_{2} \mathrm{C}_{6} \mathrm{H}_{4} \mathrm{CONH}\right]^{-}$; $279 / 281 \quad(13.1 / 12.0) \quad\left[{ }^{35} \mathrm{ClC}_{6} \mathrm{H}_{4} \mathrm{SO}_{2} \mathrm{C}_{6} \mathrm{H}_{4} \mathrm{CO}\right]^{-} /$ $\left[{ }^{37} \mathrm{ClC}_{6} \mathrm{H}_{4} \mathrm{SO}_{2} \mathrm{C}_{6} \mathrm{H}_{4} \mathrm{CO}\right]^{-} ; \quad 251 / 253 \quad(100, \quad \mathrm{BP})$ $\left[{ }^{35} \mathrm{ClC}_{6} \mathrm{H}_{4} \mathrm{SO}_{2} \mathrm{C}_{6} \mathrm{H}_{4}\right]^{-} /\left[{ }^{37} \mathrm{ClC}_{6} \mathrm{H}_{4} \mathrm{SO}_{2} \mathrm{C}_{6} \mathrm{H}_{4}\right]^{-} ; 175 / 177$ (10.3/10.2) $\left[{ }^{35} \mathrm{ClC}_{6} \mathrm{H}_{4} \mathrm{SO}_{2}\right]^{-} /\left[{ }^{37} \mathrm{ClC}_{6} \mathrm{H}_{4} \mathrm{SO}_{2}\right]^{-}$.

RP-HPLC $\left(\mathrm{CH}_{3} \mathrm{OH}: \mathrm{H}_{2} \mathrm{O}=30: 70,1 \mathrm{~mL} / \mathrm{min}, 250 \mathrm{~nm}\right)$ : purity $=99.05 \% ; t_{\mathrm{R}}=4.48 \mathrm{~min}$.

Anal. (\%): Calcd. for $\mathrm{C}_{22} \mathrm{H}_{18} \mathrm{ClNO}_{5} \mathrm{~S}(443.90 \mathrm{~g} / \mathrm{mol})$ : C, 59.53; H, 4.09; N, 3.16; S, 7.22. Found: C, 59.59; H, 4.08; N, 3.16; S, 7.24.

Synthesis of 4-benzyl-2-[4-(4-chlorophenylsulfonyl)phenyl]oxazol-5(4H)-one 4

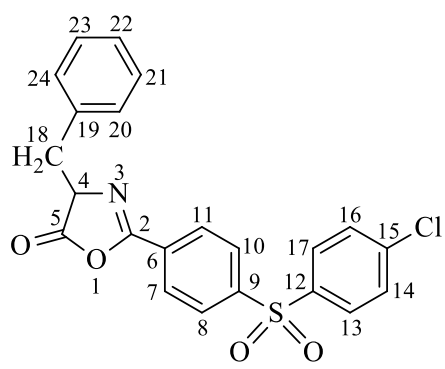

Figure 3.

Compound 4 structure with atomic numbering (for NMR assignments)

Method 1. 4-Methylmorpholine (1.15 mL, $10.5 \mathrm{mmol})$ was added under agitation into a suspension of 2-[4(4-chlorophenylsulfonyl)benzamido]-3-phenyl-

propanoic acid 3 (4.66 g, $10.5 \mathrm{mmol})$ in anhydrous dichloromethane $(50 \mathrm{~mL})$. Ethyl chloroformate $(1 \mathrm{~mL}$, $10.5 \mathrm{mmol}$ ) was added slowly to the reaction mixture. The solution obtained was stirred for another 30 min and then poured over ice water $(100 \mathrm{~mL})$. The organic phase was isolated, washed with $5 \%$ sodium hydrogen carbonate solution, then with water and dried $\left(\mathrm{MgSO}_{4}\right)$. The solvent was removed under vacuum and the solid product was recrystallized from cyclohexane as white crystals; yield $=93 \%$ (4.16 g).

Method 2. $N$-Acyl- $\alpha$-amino acid 3 (2.23 g, $5 \mathrm{mmol})$ in an 8-fold molar excess of acetic anhydride $(3.8 \mathrm{~mL}$, $40 \mathrm{mmol}$ ) was heated under stirring at $140^{\circ} \mathrm{C}$ for $1 \mathrm{~h}$ until all crystals were dissolved and then for another 30 min until saturated azlactone $\mathbf{4}$ was crystallized. After cooling, the precipitate was filtered, washed with cold ethanol and dried. White crystals were obtained; yield $=93 \%(2.06 \mathrm{~g})$.

m.p. $=170-171^{\circ} \mathrm{C}$ (cyclohexane).

$\mathrm{UV}-\mathrm{Vis}\left(\mathrm{CH}_{3} \mathrm{OH}, \lambda \mathrm{nm}\right)$ (lg $\left.\varepsilon\right)$ : 202.6 (4.48); 250.2 (4.16).

FT-IR (KBr, $\left.v \mathrm{~cm}^{-1}\right): 3094 \mathrm{~m} ; 3064 \mathrm{~m} ; 3024 \mathrm{~m} ; 2953 \mathrm{~m}$; $2923 \mathrm{w} ; 2851 \mathrm{w} ; 1825 \mathrm{vs} ; 1650 \mathrm{vs} ; 1599 \mathrm{~m} ; 1572 \mathrm{~s}$; $1493 \mathrm{~m} ; 1478 \mathrm{~m} ; 1453 \mathrm{~m} ; 1320 \mathrm{vs} ; 1296 \mathrm{vs} ; 1234 \mathrm{~m}$; $1154 \mathrm{vs} ; 1043 \mathrm{vs} ; 854 \mathrm{~m}$; 766vs.

${ }^{1} \mathrm{H}-\mathrm{NMR}\left(\mathrm{CDCl}_{3}, \delta \mathrm{ppm}, J \mathrm{~Hz}\right): 3.19$ (dd, 14.0, 6.6, 1H, H-18); 3.38 (dd, 14.0, 5.0, 1H, H-18); 4.72 (dd, 6.6, 5.0, 1H, H-4); 7.15-7.27 (m, 5H, H-20, H-21, H-22, H-23, H-24); 7.50 (d, 8.8, 2H, H-14, H-16); 7.89 (d, 8.8, 2H, H-13, H-17); 7.99 (d, 8.8, 2H, H-8, H-10); 8.05 (d, 8.8, 2H, H-7, H-11).

${ }^{13} \mathrm{C}-\mathrm{NMR}\left(\mathrm{CDCl}_{3}, \delta \mathrm{ppm}\right): 37.26(\mathrm{C}-18) ; 66.81(\mathrm{C}-4)$; 127.44 (C-22); 128.09 (C-8, C-10); 128.56 (C-20, C-24); 128.88 (C-7, C-11); 129.43 (C-13, C-17); 129.59 (C-21, C-23); 129.94 (C-14, C-16); 130.40 (C-6); 134.33 (C-19); 139.33 (C-15); 140.65 (C-9); 145.01 (C-12); 160.36 (C-2); 176.70 (C-5).

+ ESI-MS/MS ( $m / z$, rel. abund. \%): $426\left({ }^{35} \mathrm{Cl}\right) / 428\left({ }^{37} \mathrm{Cl}\right)$ $[\mathrm{M}+\mathrm{H}]^{+} ; 398 / 400$ (100, BP) [M+H-CO ${ }^{+} ; 279 / 281$ (41.5/ 21.4) $\left[{ }^{35} \mathrm{ClC}_{6} \mathrm{H}_{4} \mathrm{SO}_{2} \mathrm{C}_{6} \mathrm{H}_{4} \mathrm{CO}\right]^{+} /\left[{ }^{37} \mathrm{ClC}_{6} \mathrm{H}_{4} \mathrm{SO}_{2} \mathrm{C}_{6} \mathrm{H}_{4} \mathrm{CO}\right]^{+}$; $159 / 161\left[{ }^{35} \mathrm{ClC}_{6} \mathrm{H}_{4} \mathrm{SO}^{+} /\left[{ }^{37} \mathrm{ClC}_{6} \mathrm{H}_{4} \mathrm{SO}\right]^{+}\right.$.

GC-EI-MS ( $\mathrm{m} / \mathrm{z}$, rel. abund. \%): $381\left({ }^{35} \mathrm{Cl}\right) / 383$ $\left({ }^{37} \mathrm{Cl}\right)(8.51 / 4.26)\left[\mathrm{M}-\mathrm{CO}_{2}\right]^{+} ; 265 / 267$ (19.15/7.23) $\left[{ }^{35} \mathrm{ClC}_{6} \mathrm{H}_{4} \mathrm{SO}_{2} \mathrm{C}_{6} \mathrm{H}_{4} \mathrm{CH}_{2}\right]^{+} /\left[{ }^{37} \mathrm{ClC}_{6} \mathrm{H}_{4} \mathrm{SO}_{2} \mathrm{C}_{6} \mathrm{H}_{4} \mathrm{CH}_{2}\right]^{+}$; 159/161 (100, BP/30.64) $\left[{ }^{35} \mathrm{ClC}_{6} \mathrm{H}_{4} \mathrm{SO}^{+} /\left[{ }^{37} \mathrm{ClC}_{6} \mathrm{H}_{4} \mathrm{SO}\right]^{+}\right.$; 131 (11.91) $\left[\mathrm{C}_{6} \mathrm{H}_{5} \mathrm{CH}=\mathrm{CHCO}\right]^{+} ; 116 \quad$ (23.83) $\left[\mathrm{C}_{6} \mathrm{H}_{5} \mathrm{C}=\mathrm{C}=\mathrm{NH}\right]^{+} ; 111 / 113(8.94 / 2.55)\left[{ }^{35} \mathrm{ClC}_{6} \mathrm{H}_{4}\right]^{+} /$ $\left[{ }^{37} \mathrm{ClC}_{6} \mathrm{H}_{4}\right]^{+} ; 91$ (1.70) $\left[\mathrm{C}_{6} \mathrm{H}_{5} \mathrm{CH}_{2}\right]^{+} ; 89$ (15.32); 75 (3.40); 63 (2.55) $\left[\mathrm{C}_{5} \mathrm{H}_{3}\right]^{+} ; t_{\mathrm{R}}=46.61 \mathrm{~min}$.

RP-HPLC $\left(\mathrm{CH}_{3} \mathrm{OH}: \mathrm{H}_{2} \mathrm{O}=60: 40,1 \mathrm{~mL} / \mathrm{min}, 250 \mathrm{~nm}\right)$ : purity $=91.49 \% ; t_{\mathrm{R}}=5.05 \mathrm{~min}$.

Anal. (\%): Calcd. for $\mathrm{C}_{22} \mathrm{H}_{16} \mathrm{ClNO}_{4} \mathrm{~S}$ (425.88 g/mol): C, 62.04; H, 3.79; N, 3.29; S, 7.53. Found: C, 62.09; H, 3.78; N, 3.30; S, 7.50.

Synthesis of 2-[4-(4-chlorophenylsulfonyl)benzamido]3-phenylpropanoyl chloride 5

2-[4-(4-Chlorophenylsulfonyl)benzamido]-3-phenylpropanoic acid 3 (2.44 g, $5.5 \mathrm{mmol})$ was heated under reflux with a 25 -fold molar excess of thionyl dichloride $(10 \mathrm{~mL})$ until the emissions of gaseous hydrogen chloride and sulphur dioxide ceased. Excess $\mathrm{SOCl}_{2}$ was distilled under vacuum. Crude product in the form of yellow crystals was subsequently used without further purification; yield $=99 \%$ (2.52 g); m.p. $=90$ $92^{\circ} \mathrm{C}$.

FT-IR (KBr, $v \mathrm{~cm}^{-1}$ ): 3373m; 3090m; 3065m; 3033m; 2969w; 2949w; 2843w; 1824s; 1793s; 1651s; 1580m; 1519m; 1477s; 1455m; 1327vs; 1292s; 1161vs; 891m; $851 \mathrm{~m} ; 765 \mathrm{vs}$. 
FARMACIA, 2021, Vol. 69, 2

Synthesis of N-(1-aryl-1-oxo-3-phenylpropan-2-yl)4-(4-chlorophenylsulfonyl)benzamides $\mathbf{6 a - c}$

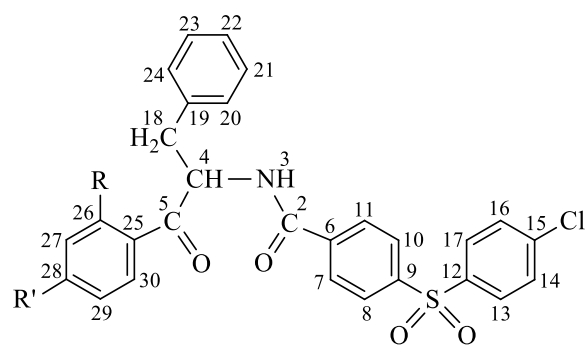

6a: R, R'=H; 6b: R=H, R' = $\mathrm{CH}_{3} ; 6 \mathrm{c}: \mathrm{R}, \mathrm{R}^{\prime}=\mathrm{CH}_{3}$

Figure 4.

General structure of compounds $\mathbf{6}$ with atomic numbering (for NMR assignments)

General method 1 for obtaining 6a-c. A 3-fold molar excess of anhydrous aluminium chloride $(2.00 \mathrm{~g}, 15$ $\mathrm{mmol})$ was gradually added under agitation to crude 4-benzyl-2-[4-(4-chlorophenylsulfonyl)phenyl]oxazol-5(4H)-one 4 (2.13 g, $5 \mathrm{mmol})$ in $25 \mathrm{~mL}$ of anhydrous aromatic hydrocarbons (benzene, toluene or $m$-xylene). The reaction mass was stirred for $20 \mathrm{~h}$ until hydrogen acid gas evolution ceased and then poured over $100 \mathrm{~mL}$ of ice-water mixture acidulated with $37 \%$ hydrochloric acid $(5 \mathrm{~mL})$. The precipitate was filtered, washed with cold water and then with a cool mixture in equal volumes of water-ethanol. The aqueous phase was separated and extracted twice with dichloromethane $(15 \mathrm{~mL})$. Combined organic layers were washed with water, dried $\left(\mathrm{Na}_{2} \mathrm{SO}_{4}\right)$ and concentrated in vacuo, leaving the second fraction of crude product. Purification by recrystallization from ethanol led to 6b,c as colourless crystals; 6a could not be isolated in the pure state.

Method 2 for obtaining 6a. A 3-fold molar excess of anhydrous $\mathrm{AlCl}_{3}(2.00 \mathrm{~g}, 15 \mathrm{mmol})$ was slowly added under stirring, to crude 2-[4-(4-chlorophenylsulfonyl)benzamido]-3-phenylpropanoyl chloride 5 (2.31 g, $5 \mathrm{mmol})$ in anhydrous benzene $(25 \mathrm{~mL}, 280 \mathrm{mmol})$. The reaction mixture was agitated for $20 \mathrm{~h}$ and then poured over ice water $(100 \mathrm{~mL})$, acidulated with fuming hydrochloric acid $(5 \mathrm{~mL})$. After extraction in dichloromethane, the organic phase was washed with a 5\% sodium hydrogen carbonate solution, then with water and dried $\left(\mathrm{Na}_{2} \mathrm{SO}_{4}\right)$. The concentration of solvents mixture in vacuo resulted in a crude product, which could not be purified.

4-(4-Chlorophenylsulfonyl)-N-(1-oxo-3-phenyl-1-ptolylpropan-2-yl)benzamide $\boldsymbol{\sigma} \boldsymbol{b}$, obtained by reaction with toluene; method 1: yield $=89 \%$ (2.31 g); m.p. = $207-208^{\circ} \mathrm{C}$ (ethanol).

$\mathrm{UV}-\mathrm{Vis}\left(\mathrm{CH}_{3} \mathrm{OH}, \lambda \mathrm{nm}\right)(\mathrm{lg} \varepsilon): 203.5$ (4.49); 253.7 (4.22).

FT-IR (KBr, $\left.v \mathrm{~cm}^{-1}\right)$ : 3393s; 3085w; 3061w; 3029m; 2970w; 2933m; 2863w; 1682s; 1650vs; 1604s; 1575m; 1513vs; 1476s; 1456m; 1329vs; 1299s; 1162vs; 855m; 753vs.

${ }^{1} \mathrm{H}-\mathrm{NMR}$ (DMSO-d 6 , $\delta$ ppm, $J \mathrm{~Hz}$ ): 2.34 (s, 3H, $\mathrm{CH}_{3}$ ); 3.02 (dd, 14.0, 9.9, 1H, H-18); 3.20 (dd, 14.0, 4.7, 1H, $\mathrm{H}-18$ ); 5.67 (m, 1H, H-4); 7.15 (bt, 7.5, 1H, H-22); 7.24 (t, 7.5, 2H, H-21, H-23); 7.25 (m, 2H, H-20, H24); 7.27 (d, 8.2, 2H, H-27, H-29); 7.69 (d, 8.5, 2H, H-14, H-16); 7.93 (d, 8.2, 4H, H-8, H-10, H-26, H30); 7.98 (d, 8.5, 2H, H-13, H-17); 8.04 (d, 8.2, 2H, $\mathrm{H}-7, \mathrm{H}-11)$; 9.21 (d, 8.0, 1H, NH).

${ }^{13} \mathrm{C}-\mathrm{NMR}\left(\mathrm{DMSO}-\mathrm{d}_{6}, \delta \mathrm{ppm}\right): 21.11\left(\mathrm{CH}_{3}\right) ; 35.97(\mathrm{C}-$ 18); 55.69 (C-4); 126.31 (C-22); 127.57 (C-8, C-10); 128.12 (C-21, C-23); 128.34 (C-7, C-11); 128.66 (C27, C-29); 129.17 (C-26, C-30); 129.32 (C-20, C24); 129.41 (C-13, C-17); 129.94 (C-14, C-16); 132.46 (C-25); 137.83 (C-19); 138.44 (C-6); 139.07 (C-15); 139.36 (C-9); 142.84 (C-12); 143.88 (C-28); 164.66 (C-2); 197.47 (C-5).

+ ESI-MS/MS $\left(\mathrm{m} / \mathrm{z}\right.$, rel. abund. \%): $518\left({ }^{35} \mathrm{Cl}\right) / 520$ $\left({ }^{37} \mathrm{Cl}\right)[\mathrm{M}+\mathrm{H}]^{+} ; 500 / 502(16.2 / 50.9)\left[\mathrm{M}+\mathrm{H}-\mathrm{H}_{2} \mathrm{O}\right]^{+}$; 296/298 (26.2/39.0) $\left[{ }^{35} \mathrm{ClC}_{6} \mathrm{H}_{4} \mathrm{SO}_{2} \mathrm{C}_{6} \mathrm{H}_{4} \mathrm{CONH}_{2}+\right.$ $\mathrm{H}]^{+} /\left[{ }^{37} \mathrm{ClC}_{6} \mathrm{H}_{4} \mathrm{SO}_{2} \mathrm{C}_{6} \mathrm{H}_{4} \mathrm{CONH}_{2}+\mathrm{H}^{+} ; 279 / 281\right.$ (55.0/ 63.4) $\left[{ }^{35} \mathrm{ClC}_{6} \mathrm{H}_{4} \mathrm{SO}_{2} \mathrm{C}_{6} \mathrm{H}_{4} \mathrm{CO}\right]^{+} /\left[{ }^{37} \mathrm{ClC}_{6} \mathrm{H}_{4} \mathrm{SO}_{2} \mathrm{C}_{6} \mathrm{H}_{4} \mathrm{CO}\right]^{+}$; 223 (100, BP) $\left[\mathrm{C}_{6} \mathrm{H}_{5} \mathrm{CH}_{2} \mathrm{CHCOC}_{6} \mathrm{H}_{4} \mathrm{CH}_{3}\right]^{+} ; 195$ (55.0/ 52.1) $\quad\left[\mathrm{C}_{6} \mathrm{H}_{5} \mathrm{CH}_{2} \mathrm{CHCOC}_{6} \mathrm{H}_{4} \mathrm{CH}_{3}-\mathrm{CO}\right]^{+} ; \quad 131$ $\left[\mathrm{C}_{6} \mathrm{H}_{5} \mathrm{CH}_{2} \mathrm{C}=\mathrm{CO}\right]^{+} ; 91(78.1 / 63.2)\left[\mathrm{CH}_{3} \mathrm{C}_{6} \mathrm{H}_{4}\right]^{+}$. RP-HPLC $\left(\mathrm{CH}_{3} \mathrm{OH}: \mathrm{H}_{2} \mathrm{O}=60: 40,1 \mathrm{~mL} / \mathrm{min}, 250 \mathrm{~nm}\right)$ : purity $=98.30 \% ; t_{\mathrm{R}}=6.40 \mathrm{~min}$.

Anal. (\%): Calcd. for $\mathrm{C}_{29} \mathrm{H}_{24} \mathrm{ClNO}_{4} \mathrm{~S}(518.02 \mathrm{~g} / \mathrm{mol})$ : C, 67.24; H, 4.67; N, 2.70; S, 6.19. Found: C, 67.29; $\mathrm{H}, 4.67 ; \mathrm{N}, 2.71 ; \mathrm{S}, 6.17$.

4-(4-Chlorophenylsulfonyl)-N-[1-(2,4-dimethylphenyl)1-oxo-3-phenylpropan-2-yl]benzamide $\mathbf{6 c}$, obtained by reaction with $m$-xylene; method 1 : yield $=91 \%$ (2.42 g); m.p. $=208-210^{\circ} \mathrm{C}$ (ethanol).

$\mathrm{UV}-\mathrm{Vis}\left(\mathrm{CH}_{3} \mathrm{OH}, \lambda \mathrm{nm}\right)$ (lg $\left.\varepsilon\right)$ : 203.5 (4.48); 252.9 (4.17).

FT-IR (KBr, $\left.v \mathrm{~cm}^{-1}\right): 3395 \mathrm{~s} ; 3087 \mathrm{~m} ; 3065 \mathrm{w} ; 3056 \mathrm{w}$; 3032w; 2963w; 2926w; 2863w; 1682s; 1654vs; 1611m; 1568m; 1512s; 1480s; 1454m; 1326vs; 1294s; 1162vs; $834 \mathrm{~m} ; 753 \mathrm{~s}$.

${ }^{1} \mathrm{H}-\mathrm{NMR}$ (DMSO-d $\mathrm{d}_{6}, \delta$ ppm, $J \mathrm{~Hz}$ ): $2.28\left(\mathrm{~s}, 3 \mathrm{H}, \mathrm{CH}_{3}\right.$ ); 2.36 (s, 3H, $\mathrm{CH}_{3}$ ); 2.99 (dd, 14.0, 9.9, 1H, H-18); 3.15 (dd, 14.0, 4.7, 1H, H-18); 5.44 (m, 1H, H-4); 7.05 7.32 (m, 7H, H-20, H-21, H-22, H-23, H-24, H-27, H-29); 7.70 (d, 8.8, 2H, H-14, H-16); 7.76 (d, 8.5, 1H, H-30); 7.84 (d, 8.8, 2H, H-8, H-10); 7.98 (d, 8.8, 2H, H-13, H-17); 8.04 (d, 8.8, 2H, H-7, H-11); 9.20 (d, 8.0, 1H, NH).

${ }^{13} \mathrm{C}-\mathrm{NMR}$ (DMSO-d $\left.6, \delta \mathrm{ppm}\right): 20.32\left(\mathrm{CH}_{3}\right) ; 20.86$ $\left(\mathrm{CH}_{3}\right)$; 35.37 (C-18); $58.12(\mathrm{C}-4) ; 126.14$ (C-29); 126.29 (C-22); 127.61 (C-8, C-10); 128.15 (C-21, C-23); 128.40 (C-30); 128.60 (C-7, C-11); 129.05 (C-20, C-24); 129.44 (C-13, C-17); 129.97 (C-14, C-16); 132.29 (C-27); 133.36 (C-25); 137.92 (C26); 138.00 (C-19); 138.50 (C-6); 139.10 (C-15); 139.37 (C-9); 141.39 (C-28); 142.84 (C-12); 164.82 (C-2); 201.35 (C-5). 
FARMACIA, 2021, Vol. 69, 2

RP-HPLC $\left(\mathrm{CH}_{3} \mathrm{OH}: \mathrm{H}_{2} \mathrm{O}=60: 40,1 \mathrm{~mL} / \mathrm{min}, 250 \mathrm{~nm}\right)$ : purity $=90.83 \% ; t_{\mathrm{R}}=7.63 \mathrm{~min}$.

Anal. (\%): Calcd. for $\mathrm{C}_{30} \mathrm{H}_{26} \mathrm{ClNO}_{4} \mathrm{~S}(532.05 \mathrm{~g} / \mathrm{mol})$ : C, 67.72; H, 4.93; N, 2.63; S, 6.03. Found: C, 67.70; H, 4.92; N, 2.64; S, 6.05 .

Synthesis of 5-aryl-4-benzyl-2-[4-(4-chlorophenylsulfonyl)phenyl]oxazoles $7 a-c$

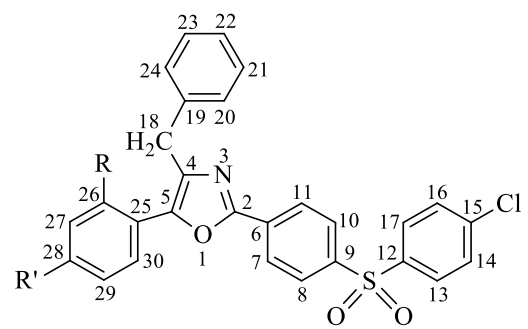

7a: $\mathrm{R}, \mathrm{R}^{\prime}=\mathrm{H} ; \mathbf{7 b}: \mathrm{R}=\mathrm{H}, \mathrm{R}^{\prime}=\mathrm{CH}_{3} ; \mathbf{7 c}: \mathrm{R}, \mathrm{R}^{\prime}=\mathrm{CH}_{3}$

Figure 5.

General structure of compounds 7 with atomic numbering (for NMR assignments)

General method 1 for obtaining 7a-c. Crude $N$-(1aryl-1-oxo-3-phenylpropan-2-yl)-4-(4-chlorophenylsulfonyl)benzamides 6a-c $(10 \mathrm{mmol})$ in phosphoryl trichloride $(20 \mathrm{~mL}, 217.83 \mathrm{mmol})$ were refluxed for $4 \mathrm{~h}$. Unreacted $\mathrm{POCl}_{3}$ was removed by distillation under reduced pressure. After cooling, oily residue was added into ice-water and extracted twice with dichloromethane $(20 \mathrm{~mL})$. Combined organic phases were washed several times with $5 \% \mathrm{NaHCO}_{3}$ solution, then with water and dried $\left(\mathrm{Na}_{2} \mathrm{SO}_{4}\right)$. After solvent removal, crude products $\mathbf{7 a - c}$ were purified by recrystallization from ethanol as colourless crystals. Method 2 for obtaining $7 \boldsymbol{b}$. 4-(4-Chlorophenylsulfonyl)$N$-(1-oxo-3-phenyl-1-p-tolylpropan-2-yl)benzamide 6b $(5.444 \mathrm{~g}, 10.51 \mathrm{mmol})$ was dissolved in ethyl acetate $(40 \mathrm{~mL})$. Acetic anhydride $(3 \mathrm{~mL}, 3.22 \mathrm{~g}, 31.54$ $\mathrm{mmol})$ and $98 \%$ sulfuric acid $(0.17 \mathrm{~mL}, 0.31 \mathrm{~g}, 3.16$ $\mathrm{mmol})$ in ethyl acetate $(2.5 \mathrm{~mL})$ were added. After refluxing for $3 \mathrm{~h}$, a $5 \mathrm{~N} \mathrm{NaOH}$ solution $(12.6 \mathrm{~mL}$, $63 \mathrm{mmol}$ ) diluted to $25 \mathrm{~mL}$ with water was added at room temperature. The reaction mass was heated for another $30 \mathrm{~min}$ and then cooled. The precipitate was filtered, washed with cold $1 \mathrm{~N} \mathrm{HCl}, 10 \% \mathrm{NaCl}$ solution and water. Layers of the filtrate were separated and the organic phase was dried $\left(\mathrm{Na}_{2} \mathrm{SO}_{4}\right)$, and concentrated to dryness in vacuo, leaving the second fraction of crude product. High purity colourless crystals of compound $\mathbf{7 b}$ resulted after purification.

4-Benzyl-2-[4-(4-chlorophenylsulfonyl)phenyl]-5phenyloxazole $7 a$

Method 1: yield $=89 \%$ (4.33 g); m.p. $=199-201^{\circ} \mathrm{C}$ (ethanol).

UV-Vis $\left(\mathrm{CH}_{3} \mathrm{OH}, \lambda \mathrm{nm}\right)(\lg \varepsilon): 203.5$ (4.48); 247.6 (4.06); 333.9 (4.11).

FT-IR $\left(\mathrm{KBr}, v \mathrm{~cm}^{-1}\right)$ : 3087m; 3061m; 3028m; 2926w; $2845 \mathrm{w} ; 1602 \mathrm{~s} ; 1590 \mathrm{~s} ; 1547 \mathrm{~m} ; 1495 \mathrm{~s} ; 1476 \mathrm{~s} ; 1453 \mathrm{~m}$; $1327 \mathrm{vs} ; 1290 \mathrm{~s} ; 1281 \mathrm{~s} ; 1158 \mathrm{vs} ; 1088 \mathrm{vs} ; 845 \mathrm{~s} ; 765 \mathrm{vs}$.
${ }^{1} \mathrm{H}-\mathrm{NMR}\left(\mathrm{CDCl}_{3}, \delta \mathrm{ppm}, J \mathrm{~Hz}\right): 4.20$ (s, 2H, H-18); 7.18 - 7.32 (m, 5H, H-20, H-21, H-22, H-23, H-24); 7.36 (bt, 7.9, 1H, H-28); 7.45 (bt, 7.9, 2H, H-27, H29); 7.49 (d, 8.8, 2H, H-14, H-16); 7.68 (d, 7.9, 2H, H-26, H-30); 7.85 (d, 8.8, 2H, H-13, H-17); 7.95 (d, 8.8, 2H, H-8, H-10); 8.16 (d, 8.8, 2H, H-7, H-11).

${ }^{13} \mathrm{C}-\mathrm{NMR}\left(\mathrm{CDCl}_{3}, \delta \mathrm{ppm}\right): 33.18(\mathrm{C}-18) ; 126.00(\mathrm{C}-$ 26, C-30); 126.68 (C-22); 127.18 (C-8, C-10); 128.33 (C-7, C-11); 128.51 (C-20, C-24); 128.60 (C-28); 128.78 (C-21, C-23); 129.11 (C-27, C-29); 129.30 (C-13, C-17); 129.88 (C-14, C-16); 132.00 (C-25); 136.74 (C-6); 138.34 (C-4, C-19); 139.98 (C-9); 140.31 (C-15); 142.17 (C-12); 147.99 (C-5); 158.00 (C-2). RP-HPLC $\left(\mathrm{CH}_{3} \mathrm{OH}: \mathrm{H}_{2} \mathrm{O}=70: 30,1 \mathrm{~mL} / \mathrm{min}, 335 \mathrm{~nm}\right)$ : purity $=95.13 \% ; t_{\mathrm{R}}=4.68 \mathrm{~min}$.

Anal. (\%): Calcd. for $\mathrm{C}_{28} \mathrm{H}_{20} \mathrm{ClNO}_{3} \mathrm{~S}(485.98 \mathrm{~g} / \mathrm{mol})$ : C, 69.20; H, 4.15; N, 2.88; S, 6.60. Found: C, 69.25; $\mathrm{H}, 4.14 ; \mathrm{N}, 2.89 ; \mathrm{S}, 6.58$.

4-Benzyl-2-[4-(4-chlorophenylsulfonyl)phenyl]-5p-tolyloxazole $7 \boldsymbol{b}$

Method 1: yield $=95 \%(4.75 \mathrm{~g})$, method $2:$ yield $=$ $90 \%(4.73 \mathrm{~g}) ;$ m.p. $=213-214^{\circ} \mathrm{C}$ (ethanol).

$\mathrm{UV}-\mathrm{Vis}\left(\mathrm{CH}_{3} \mathrm{OH}, \lambda \mathrm{nm}\right)$ (lg $\left.\varepsilon\right)$ : 203.5 (4.49); 250.2 (4.10); 340.1 (4.12).

FT-IR (KBr, $\left.v \mathrm{~cm}^{-1}\right): 3089 \mathrm{~m} ; 3063 \mathrm{~m} ; 3029 \mathrm{~m} ; 2919 \mathrm{~m}$; 2861w; 1595s; 1544m; 1509s; 1495s; 1476s; 1454m; 1326vs; 1290s; 1283s; 1155vs; 1087vs; 843s; 767vs.

${ }^{1} \mathrm{H}-\mathrm{NMR}\left(\mathrm{CDCl}_{3}, \delta \mathrm{ppm}, J \mathrm{~Hz}\right): 2.34\left(\mathrm{~s}, 3 \mathrm{H}, \mathrm{CH}_{3}\right)$; 4.13 (s, 2H, H-18); 7.15-7.28 (m, 7H, H-20, H-21, H-22, H-23, H-24, H-27, H-29); 7.50 (d, 7.9, 2H, H-26, H-30); 7.64 (d, 8.8, 2H, H-14, H-16); 7.85 (d, 8.8, 2H, H-13, H-17); 7.95 (d, 8.8, 2H, H-8, H-10); 8.16 (d, 8.8, 2H, H-7, H-11).

${ }^{13} \mathrm{C}-\mathrm{NMR}\left(\mathrm{CDCl}_{3}, \delta \mathrm{ppm}\right): 21.43\left(\mathrm{CH}_{3}\right) ; 33.17(\mathrm{C}-$ 18); 125.49 (C-6); 125.94 (C-26, C-30); 126.60 (C22); 127.08 (C-8, C-10); 128.28 (C-7, C-11); 128.50 (C-20, C-24); 128.72 (C-21, C-23); 129.26 (C-13, C-17); 129.77 (C-27, C-29); 129.83 (C-14, C-16); 132.07 (C-25); 136.16 (C-28); 138.48 (C-19); 138.86 (C-4); 140.05 (C-9); 140.24 (C-12); 142.04 (C-15); 148.18 (C-5); 157.70 (C-2).

+ESI-MS/MS $\left(\mathrm{m} / \mathrm{z}\right.$, rel. abund. \%): $500\left({ }^{35} \mathrm{Cl}\right) / 502$ $\left({ }^{37} \mathrm{Cl}\right)[\mathrm{M}+\mathrm{H}]^{+} ; 422 / 424\left[\mathrm{M}+\mathrm{H}-\mathrm{C}_{6} \mathrm{H}_{6}\right]^{+} ; 279 / 281(38.0 /$ 37.1) $\left[{ }^{35} \mathrm{ClC}_{6} \mathrm{H}_{4} \mathrm{SO}_{2} \mathrm{C}_{6} \mathrm{H}_{4} \mathrm{CO}\right]^{+} /\left[{ }^{37} \mathrm{ClC}_{6} \mathrm{H}_{4} \mathrm{SO}_{2} \mathrm{C}_{6} \mathrm{H}_{4} \mathrm{CO}\right]^{+}$; $159 / 161\left[{ }^{35} \mathrm{ClC}_{6} \mathrm{H}_{4} \mathrm{SO}\right]^{+} /\left[{ }^{37} \mathrm{ClC}_{6} \mathrm{H}_{4} \mathrm{SO}\right]^{+} ; 117$ (100, BP) $\left[\mathrm{CH}_{3} \mathrm{C}_{6} \mathrm{H}_{4} \mathrm{C}=\mathrm{CH}_{2}\right]^{+}$.

RP-HPLC $\left(\mathrm{CH}_{3} \mathrm{OH}: \mathrm{H}_{2} \mathrm{O}=70: 30,1 \mathrm{~mL} / \mathrm{min}, 335 \mathrm{~nm}\right)$ : purity $=97.57 \% ; t_{\mathrm{R}}=4.85 \mathrm{~min}$.

Anal. (\%): Calcd. for $\mathrm{C}_{29} \mathrm{H}_{22} \mathrm{ClNO}_{3} \mathrm{~S}(500.01 \mathrm{~g} / \mathrm{mol})$ : C, 69.66; H, 4.43; N, 2.80; S, 6.41. Found: C, 69.69; $\mathrm{H}, 4.42 ; \mathrm{N}, 2.81 ; \mathrm{S}, 6.40$.

4-Benzyl-2-[4-(4-chlorophenylsulfonyl)phenyl]-5-

(2,4-dimethylphenyl)oxazole 7c

Method 1: yield $=90 \%(4.63 \mathrm{~g}) ;$ m.p. $=158-160^{\circ} \mathrm{C}$ (ethanol).

UV-Vis $\left(\mathrm{CH}_{3} \mathrm{OH}, \lambda \mathrm{nm}\right)$ (lg $\left.\varepsilon\right): 203.5$ (4.47); 246.7 (4.02); 322.5 (4.01). 
FARMACIA, 2021, Vol. 69, 2

FT-IR (KBr, $\left.v \mathrm{~cm}^{-1}\right)$ : 3087w; 3063w; 3027m; 2923m; 2860w; 1602s; 1582s; 1494s; 1475m; 1453m; 1327vs; 1289s; 1281s; 1158vs; 1088vs; 843m; 767vs. ${ }^{1} \mathrm{H}-\mathrm{NMR}\left(\mathrm{CDCl}_{3}, \delta \mathrm{ppm}, J \mathrm{~Hz}\right): 2.31\left(\mathrm{~s}, 3 \mathrm{H}, \mathrm{CH}_{3}\right)$; 2.37 (s, 3H, $\mathrm{CH}_{3}$ ); 3.94 (s, 2H, H-18); 7.06 (bd, 7.7, 1H, H-29); 7.10-7.30 (m, 5H, H-20, H-21, H-22, H-23, H-24); 7.14 (bs, 1H, H-27); 7.20 (d, 7.7, 1H, H-30); 7.48 (d, 8.8, 2H, H-14, H-16); 7.88 (d, 8.8, 2H, H13, H-17); 7.98 (d, 8.6, 2H, H-8, H-10); 8.17 (d, 8.6, 2H, H-7, H-11).

${ }^{13} \mathrm{C}-\mathrm{NMR}\left(\mathrm{CDCl}_{3}, \delta \mathrm{ppm}\right): 20.45\left(\mathrm{CH}_{3}\right) ; 21.41\left(\mathrm{CH}_{3}\right)$; 32.45 (C-18); 124.46 (C-6); 126.46 (C-22); 126.77 (C-29); 127.01 (C-8, C-10); 128.30 (C-7, C-11); 128.59 (C-20, C-24); 128.65 (C-21, C-23); 129.27 (C-13, C-17); 129.83 (C-14, C-16); 130.15 (C-30); 131.79 (C-27); 132.32 (C-25); 137.82 (C-26); 138.95 (C-4, C-19); 139.97 (C-9, C-28); 140.24 (C-12); 142.04 (C-15); 148.59 (C-5); 158.42 (C-2).

RP-HPLC $\left(\mathrm{CH}_{3} \mathrm{OH}: \mathrm{H}_{2} \mathrm{O}=70: 30,1 \mathrm{~mL} / \mathrm{min}, 335\right.$ $\mathrm{nm})$ : purity $=99.15 \% ; t_{\mathrm{R}}=4.87 \mathrm{~min}$.

Anal. (\%): Calcd. for $\mathrm{C}_{30} \mathrm{H}_{24} \mathrm{ClNO}_{3} \mathrm{~S}(514.03 \mathrm{~g} / \mathrm{mol})$ : C, 70.10; H, 4.71; N, 2.72; S, 6.24. Found: C, 70.15; $\mathrm{H}, 4.70 ; \mathrm{N}, 2.71 ; \mathrm{S}, 6.22$.

Cytotoxicity evaluation

The biological assessment was performed by Daphnia magna bioassay. The procedure was described in our previous works [10]. Young daphnids which were exposed to six concentrations from each compound (from 2.2 to $46 \mu \mathrm{g} / \mathrm{mL}$ ) for a period of $72 \mathrm{~h}$ were used. The bioassay was carried out in duplicate. Phenylalanine and compound $\mathbf{1}$ were used as positive controls, whereas $1 \%$ dimethyl sulfoxide as a negative control. The experiment was performed at $25 \pm 1^{\circ} \mathrm{C}$ in Sanyo MLR-351H, USA climatic chamber.

Lethality was registered at 24, 48 and $72 \mathrm{~h}$. Lethality curves were plotted using the logarithm of concentration against lethality percentage, $\mathrm{L}(\%)$. Prediction of $\mathrm{LC}_{5048 \mathrm{~h}}$ values was performed using GUSAR software.

\section{Results and Discussion}

\section{Chemistry}

Synthetic approach to title compounds starts from the key precursor, 4-(4-chlorophenylsulfonyl)-benzoic acid $\mathbf{1}$, and corresponding acyl chloride $\mathbf{2}$ which were previously reported in the literature [34, 35].

Compound 1 was synthesized in two stages: by FriedelCrafts sulfonylation of chlorobenzene with 4-methylbenzenesulfonyl chloride in presence of $\mathrm{AlCl}_{3}$, followed by oxidation of 1-(4-chlorophenylsulfonyl)4-methylbenzene with chromium trioxide in glacial acetic acid, both reactions occurring at reflux [34]. Then, carboxylic acid $\mathbf{1}$ was transformed by reaction with thionyl dichloride into 4-(4-chlorophenylsulfonyl)benzoyl chloride 2 [33]. This crude product was used for the $\mathrm{N}$-acylation of phenylalanine to 2-[4-(4-chlorophenylsulfonyl)benzamido]-3-phenylpropanoic acid $\mathbf{3}$.
Intramolecular cyclodehydration of compound $\mathbf{3}$, using ethyl chloroformate in presence of $\mathrm{N}$-methylmorpholine in $\mathrm{CH}_{2} \mathrm{Cl}_{2}$ at ambient temperature or acetic anhydride at reflux, led to 4-benzyl-2-[4-(4-chlorophenylsulfonyl)phenyl]oxazol-5(4H)-one 4. Cyclization under basic conditions can be explained by a reaction mechanism similar to that previously indicated by us in literature for another 1,3-oxazol-5(4H)-one [13]. Cyclization of $\mathbf{3}$ to saturated azlactone $\mathbf{4}$ in the presence of acetic anhydride can take place through the reaction mechanism proposed in Figure 6. $N$-Acyl- $\alpha$-amino acid $\mathbf{3}$ is in equilibrium with its enol tautomer $\mathbf{I}$, which under the action of acetic anhydride gives a nucleophilic substitution reaction, with the formation of key intermediate, namely unstable acyclic ester II, which by an intramolecular nucleophilic addition mechanism with the removal of acetic acid leads to pentatomic heterocyclic compound 4.

Also, $\mathrm{N}$-acylated phenylalanine $\mathbf{3}$ was reacted with $\mathrm{SOCl}_{2}$ at reflux to 2-[4-(4-chlorophenylsulfonyl)benzamido]-3-phenylpropanoyl chloride $\mathbf{5}$ by a nucleophilic substitution mechanism.

Friedel-Crafts acylation reaction of arenes (benzene, toluene, $m$-xylene) with 1,3-oxazol-5(4H)-one 4, catalysed by $\mathrm{AlCl}_{3}$, yielded $N$-(1-aryl-1-oxo-3-phenylpropan-2yl)-4-(4-chlorophenylsulfonyl)benzamides 6 by an electrophilic aromatic substitution mechanism presented by us in a formerly article [33]. $N$-Acyl- $\alpha$-amino ketones 6b,c were isolated as pure colorless crystals and physicochemically characterized, except for 4-(4chlorophenylsulfonyl)- $N$-(1-oxo-1,3-diphenylpropan2-yl)benzamide $\mathbf{6 a}$ (obtained by reaction of $\mathbf{4}$ and $\mathbf{5}$, respectively with benzene), which was used in crude form to synthesize 1,3-oxazole $\mathbf{7 a}$.

4-Aryl-2-aza-3-benzyl-1-[4-(4-chlorophenylsulfonyl)phenyl]-1,4-butanediones 6a-c underwent RobinsonGabriel cyclization using phosphoryl trichloride at reflux with the formation of 5-aryl-4-benzyl-2-[4(4-chlorophenylsulfonyl)phenyl]oxazoles 7a-c in very good yields. Also, cyclization of $\mathbf{6 a}$ to $\mathbf{7 b}$ was carried out using $\mathrm{H}_{2} \mathrm{SO}_{4} /\left(\mathrm{CH}_{3} \mathrm{CO}\right)_{2} \mathrm{O}$ in ethyl acetate, by heating under reflux. Proposed reaction mechanisms for the synthesis of 1,3-oxazole analogs starting from $N$-acyl- $\alpha$-amino ketones under the action of $\mathrm{POCl}_{3}$ and $\mathrm{H}_{2} \mathrm{SO}_{4}$, respectively are described in our recent literature [10].

Spectral analysis methods (UV-Vis, IR, MS, ${ }^{1} \mathrm{H}$ - and

${ }^{13} \mathrm{C}-\mathrm{NMR}$ ) and elemental analysis were used for characterizing synthesized compounds.

$U V$-Vis Spectral Data

UV-Vis spectra of new compounds show a sharp band (E band) at $\lambda_{\max }=202.6$ or $203.5 \mathrm{~nm}$ and an absorption maximum (B band) in range 246.7-253.7 nm. Besides, electronic absorption spectra of 1,3-oxazoles 7 presented a third intense band at longer wavelengths (322.5 $340.1 \mathrm{~nm}$ ), because of conjugation extension by the formation of the 1,3-oxazole chromophore. 


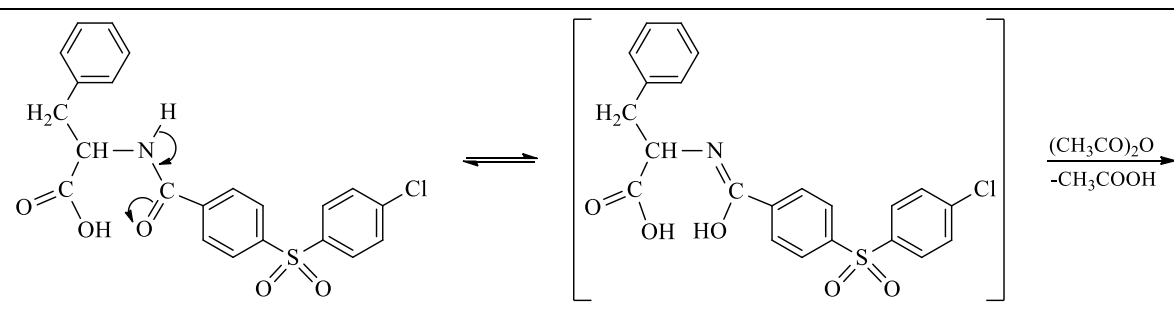

3

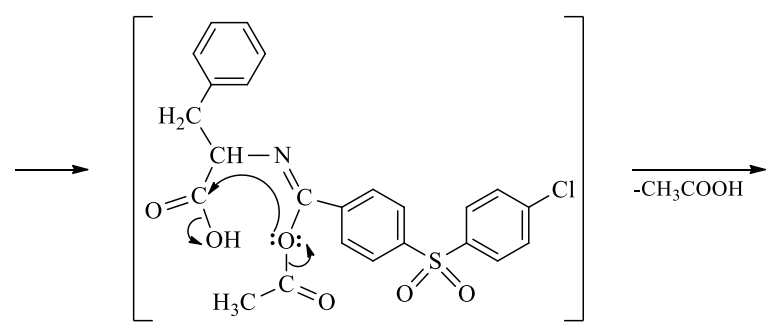

II
I<smiles>O=C1OC(c2ccc(S(=O)(=O)c3ccc(Cl)cc3)cc2)=NC1Cc1ccccc1</smiles>

4

Figure 6.

Proposed mechanism for synthesis of 1,3-oxazol-5(4H)-one 4 from $N$-acyl- $\alpha$-amino acid 3 in presence of $\left(\mathrm{CH}_{3} \mathrm{CO}\right)_{2} \mathrm{O}$

\section{IR Spectral Data}

Infrared absorption spectra of newly synthesized compounds show absorption bands at characteristically wavenumbers of structural fragments present in molecules. Evidence of formation of open-chain intermediates $\mathbf{3}$ and $\mathbf{6}$, is the presence in IR spectra of following characteristic absorption bands in intervals: $3355-3395 \mathrm{~cm}^{-1}$ due to $\mathrm{N}-\mathrm{H}$ stretching vibration, $v(\mathrm{~N}-\mathrm{H}), 1682-1734 \mathrm{~cm}^{-1}$ due to carbonyl stretching vibration, $v(\underline{\mathrm{O}=\mathrm{C}}-\mathrm{C}), 1623-1654 \mathrm{~cm}^{-1}$ due to amidic carbonyl group absorption, $v(\mathrm{O}=\mathrm{C}-\mathrm{N})$. Furthermore, for the hydrogen-bonded dimer of $\mathbf{3}$, a very broad absorption band due to $\mathrm{O}-\mathrm{H}$ stretching vibration, $v(\mathrm{O}-\mathrm{H})$, is extending from $2500 \mathrm{~cm}^{-1}$ to $3200 \mathrm{~cm}^{-1}$. Representative for dimer are also four broad medium intensity bands between 2532 and $2704 \mathrm{~cm}^{-1}$. Main absorption bands presented in IR spectrum of acyl chloride 5 (namely, two strong absorption bands due to $v(\underline{\mathrm{O}=\mathrm{C}}-\mathrm{Cl})$ at $1824 \mathrm{~cm}^{-1}$ assigned to fundamental vibration and $1793 \mathrm{~cm}^{-1}$ named Fermi resonance band, and a medium absorption band due to $v(\underline{\mathrm{Cl}}-\mathrm{C}=\mathrm{O})$ at $\left.891 \mathrm{~cm}^{-1}\right)$ confirmed that reaction of $N$-acyl- $\alpha$-amino acid 3 with $\mathrm{SOCl}_{2}$ took place. IR spectra of heterocycles $\mathbf{4}$ and $\mathbf{7}$ differ from those of acyclic precursors (3 and 6) from which they were obtained, by fact that they have absorption bands at characteristic, different values of wavenumbers, which proves that cyclizations have occurred. In IR spectrum of $\mathbf{4}$, very strong absorption band due to carbonyl valence vibration, $v(\mathrm{C}=\mathrm{O})$, is shifted at $1825 \mathrm{~cm}^{-1}$ and $v(\mathrm{~N}-$ $\mathrm{H}), v(\mathrm{O}-\mathrm{H})$ and $v(\underline{\mathrm{O}=\mathrm{C}}-\mathrm{N})$ absorption bands which are characteristic for acyclic intermediate $\mathbf{3}$ were not presented. In the case of 1,3-oxazoles 7, absorption peaks were not registered in the $\mathrm{N}-\mathrm{H}$ and $\mathrm{C}=\mathrm{O}$ regions. The absorption band at $1650 \mathrm{~cm}^{-1}$ (4) and 1595 or
$1602 \mathrm{~cm}^{-1}$ (7) was attributed to $\mathrm{C}=\mathrm{N}$ stretching vibration, $v(\mathrm{C}=\mathrm{N})$, of these five-membered-ring systems. Moreover, IR spectra of $\mathbf{4}$ and $\mathbf{7}$ showed supplementary, very strong peaks at $1043 \mathrm{~cm}^{-1}(4)$ and 1087 or $1088 \mathrm{~cm}^{-1}$ (7) due to symmetrical C-O-C stretching vibration, $v_{\text {sym }}(\mathrm{C}-\mathrm{O}-\mathrm{C})$. Absorption band due to asymmetrical stretching vibration of the C-O-C system, $v_{\text {as }}(\mathrm{C}-\mathrm{O}-\mathrm{C})$, appears in the saturated azlactone 4 spectrum at $1234 \mathrm{~cm}^{-1}$ and in 1,3-oxazoles 7a-c spectra at 1281 or $1283 \mathrm{~cm}^{-1}$.

\section{NMR Spectral Data}

Signals in the ${ }^{1} \mathrm{H}-\mathrm{NMR}$ spectra of new compounds are also consistent with proposed chemical structures. Besides, 2D ${ }^{1} \mathrm{H}-{ }^{1} \mathrm{H}$ COSY experiments were performed, which facilitated unambiguous assignments. It can be noticed presence in ${ }^{1} \mathrm{H}-\mathrm{NMR}$ spectra of acyclic intermediates $\mathbf{3}$ and $\mathbf{6}$ of a signal as a doublet at $\delta=$ $9.00 \mathrm{ppm}(3)$ and 9.20 or $9.21 \mathrm{ppm}(\mathbf{6})$, assigned to secondary amide proton (H-3), which couples with $\mathrm{H}-4\left({ }^{3} J=8.0 \mathrm{~Hz}\right)$. In ${ }^{1} \mathrm{H}-\mathrm{NMR}$ spectra of heterocyclic compounds 4 and 7 , signal attributed to the proton of NH group (H-3) from acyclic precursors 3 and $\mathbf{6}$ is absent and this demonstrates that cyclization products 4 and 7 have been synthesized. In ${ }^{1} \mathrm{H}-\mathrm{NMR}$ spectra of 3 and 6, the H-4 signal appears as a multiplet at 4.64 ppm (3) and 5.44 or 5.67 ppm (6), which results from coupling to two nonequivalent protons of $\mathrm{CH}_{2}$ group and one proton of $\mathrm{NH}$ group. For 1,3-oxazol$5(4 H)$-one 4 , the signal of methine proton from $\mathrm{C}-4$ is recorded at $4.72 \mathrm{ppm}$ as a doublet of doublets due to coupling to both nonequivalent protons of $\mathrm{CH}_{2}$ group from benzyl fragment linked in position 4 . Signal presented by $\mathrm{H}-4$ proton in ${ }^{1} \mathrm{H}-\mathrm{NMR}$ spectra of compounds $\mathbf{6}$, is absent in the case of heterocyclic compounds $\mathbf{7}$ and this is evidence that cyclization 
has taken place. Moreover, the two signals of H-18 nonequivalent protons from $\mathrm{CH}_{2}$ group as a doublet of doublets, in the ${ }^{1} \mathrm{H}-\mathrm{NMR}$ spectrum of saturated azlactone 4, showed discernible downfield shifts of 0.15 and $0.17 \mathrm{ppm}$, relative to $\mathrm{CH}_{2}$ protons signals from $N$-acyl- $\alpha$-amino acid $\mathbf{3}$ spectrum, due to stronger deshielding effect of 1,3-oxazol-5(4H)-one ring, compared to that of carboxyl and $\mathrm{N}$-monosubstituted aminocarbonyl groups from acyclic compound $\mathbf{3}$. Further proof for formation of 2,5-diaryl-4-benzyl1,3-oxazoles 7 is provided by the fact that their ${ }^{1} \mathrm{H}$ NMR spectra revealed a downfield shift of signal attributed to two $\mathrm{H}-18$ protons of $\mathrm{CH}_{2}$ group as a singlet (because in this case, the two protons of $\mathrm{CH}_{2}$ group are magnetically equivalent) in the range $3.94-4.20$ ppm compared to the two signals as a doublet of doublets (because of germinal coupling between nonequivalent protons of $\mathrm{CH}_{2}$ group with ${ }^{2} J=14.0 \mathrm{~Hz}$ and of vicinal coupling of these protons with $\mathrm{H}-4$ with ${ }^{3} J=9.9$ and $4.7 \mathrm{~Hz}$, respectively) at $\delta$ values of 2.99 or $3.02 \mathrm{ppm}$ and 3.15 or $3.20 \mathrm{ppm}$ registered for $\mathrm{N}$-acyl- $\alpha$-amino ketones $\mathbf{6}$. This is due to the stronger deshielding effect of the 1,3-oxazole ring (from 7) in comparison with that of carbonyl and $\mathrm{N}$-monosubstituted carboxamide groups from acyclic intermediates 6 . Syntheses of compounds 3, 4, 6 and 7 were also proved by ${ }^{13} \mathrm{C}$-NMR spectra. Moreover, $2 \mathrm{D}{ }^{1} \mathrm{H}-{ }^{13} \mathrm{C}$ HETCOR experiments allowed univocal assignments of ${ }^{13} \mathrm{C}$ signals. C-4 peak, which appears at $\delta=54.38 \mathrm{ppm}$ in the ${ }^{13} \mathrm{C}$-NMR spectrum of $\mathrm{N}$-acyl phenylalanine $\mathbf{3}$, is shifted downfield with 12.43 ppm after cyclization to 4 . Furthermore, in compound 4, C-2 atom resonated at $160.36 \mathrm{ppm}$ (being shielded with $4.76 \mathrm{ppm}$ than C-2 from -CONH- group of acyclic precursor 3), and C-5 at 176.70 ppm (being shifted downfield with 3.84 ppm than $\mathrm{C}-5$ from $-\mathrm{COOH}$ group of 3 ). The upfield signal assigned to $\mathrm{C}-2$ from the structure of 1,3-oxazoles 7 is present in interval 157.70-158.42 ppm, while the signal attributed to $\mathrm{C}-2$ from $\mathrm{N}$-monosubstituted carboxamide group of acyclic precursors 6 (from 164.66 or $164.82 \mathrm{ppm}$ ) is absent in case of

heterocyclic compounds 7. Furthermore, C-5 from the 1,3-oxazole nucleus (7) resonated in range 147.99 $148.59 \mathrm{ppm}$, whereas ketonic carbonyl carbon of $\mathrm{N}$ acyl- $\alpha$-amino ketones 6 at $\delta=197.47$ or 201.35 ppm revealing an upfield shift for this $\mathrm{C}$ atom in 1,3oxazoles 7 structure, which confirm that obtaining of 1,3-oxazole ring occurred.

Mass Spectral Data

A supplementary contribution in the structural elucidation of compounds $3, \mathbf{4}, \mathbf{6 b}$, and $\mathbf{7 b}$ was achieved by registering mass spectra by LC-ESI-MS/MS technique. Protonated and/or deprotonated molecular ions and main fragments of these compounds corresponding to chloride isotopes $\left({ }^{35} \mathrm{Cl} /{ }^{37} \mathrm{Cl}\right)$ are reported in the experimental part. Due to lower polarity, higher volatility and stability at high temperatures only saturated azlactone 4 could be analyzed by GC-EI-MS. In this case, the two molecular ions of $\mathbf{4}$ (corresponding to ${ }^{35} \mathrm{Cl}$ and ${ }^{37} \mathrm{Cl}$ isotopes) being very unstable does not show signals in the mass spectrum. These begin to split at the level of 1,3-oxazol-5(4H)-one ring by eliminating a molecule of $\mathrm{CO}_{2}$ when two cation-radicals corresponding to isotopes of chloride with $\mathrm{m} / \mathrm{z}=381 / 383$ (with relative abundances: $8.51 \%$ and $4.26 \%$, respectively) are formed. Base peak (PB), cation $\left[{ }^{35} \mathrm{ClC}_{6} \mathrm{H}_{4} \mathrm{SO}\right]^{+}$with $m / z=159$ and corresponding fragment $\left[{ }^{37} \mathrm{ClC}_{6} \mathrm{H}_{4} \mathrm{SO}^{+}\right.$ with $m / z=161$ (with relative abundance $=30.64 \%$ ) were obtained according to ${ }^{35} \mathrm{Cl} /{ }^{37} \mathrm{Cl}$ isotopic ratio of approximatively $3: 1$. The fragmentation pattern is consistent with structure.

Cytotoxicity evaluation

At $24 \mathrm{~h}$ tested compounds exhibited a maximum L\% of $20 \%$. At $48 \mathrm{~h}$, except for $\mathbf{7 a}$ and $\mathbf{1}$, all compounds induced $\mathrm{L} \%$ lower than $40 \%$. $\mathrm{LC}_{50}$ at $48 \mathrm{~h}$ for compound 7a was $100.1 \mu \mathrm{g} / \mathrm{mL}$ (95\% CI: $7.24-1384 \mu \mathrm{g} / \mathrm{mL}$ ), whereas for compound $\mathbf{1}$ the value couldn't be calculated due poor correlation between concentration and $\mathrm{L} \%$. For all other compounds, $\mathrm{LC}_{50}$ couldn't be calculated at 24 and $48 \mathrm{~h}$. The $\mathrm{LC}_{50}$ values at $72 \mathrm{~h}$ are presented in Table I and the lethality curves in Figure 7.

Table I

Results of Daphnia magna bioassay

\begin{tabular}{|c|c|c|c|}
\hline Tested compounds & $\begin{array}{c}\text { Predicted } \mathbf{L C}_{\mathbf{5 0}} \mathbf{4 8} \mathbf{~} \\
(\boldsymbol{\mu g} / \mathbf{m L})\end{array}$ & $\begin{array}{c}\text { Determined } \mathbf{L C}_{\mathbf{5 0} \mathbf{7 2}} \mathbf{~} \\
(\boldsymbol{\mu g} / \mathbf{m L} \mathbf{)}\end{array}$ & $\begin{array}{c}\mathbf{9 5 \%} \mathbf{C I} \text { of } \mathbf{L C}_{\mathbf{5 0}} \mathbf{7 2} \mathbf{~} \\
(\boldsymbol{\mu g} / \mathbf{m L} \mathbf{)}\end{array}$ \\
\hline $\mathbf{3}$ & 1.25 & 44.24 & $37.67-51.95$ \\
\hline $\mathbf{4}$ & 1.04 & 26.58 & $21.55-32.79$ \\
\hline $\mathbf{6 b}$ & 0.119 & 37.27 & $22.02-63.10$ \\
\hline $\mathbf{6 c}$ & 0.057 & $\approx 22.95$ & $\mathrm{NC}$ \\
\hline $\mathbf{7 a}$ & 0.287 & $\approx 17.71$ & $\mathrm{NC}$ \\
\hline $\mathbf{7 b}$ & 0.094 & 33.35 & $23.58-47.16$ \\
\hline $\mathbf{7 c}$ & 0.029 & $\approx 47.67$ & $\mathrm{NC}$ \\
\hline phenylalanine & 170.79 & $\mathrm{NC}$ & $\mathrm{NC}$ \\
\hline $\mathbf{1}$ & 40.68 & 44.88 & $28.48-70.71$ \\
\hline
\end{tabular}

$\mathrm{LC}_{50}-50 \%$ lethal concentration; $95 \% \mathrm{CI}-95 \%$ confidence interval; $\mathrm{NC}-$ not calculated due to obtained results 


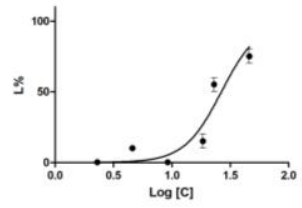

a

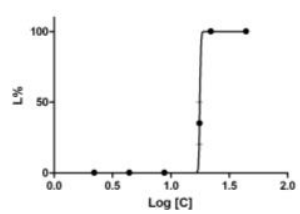

d

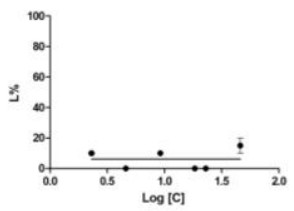

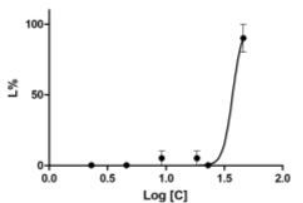

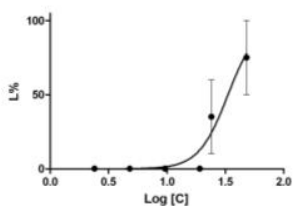

e

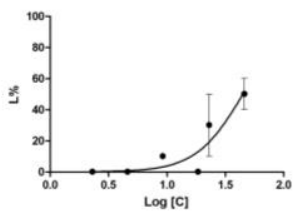

$\mathrm{h}$

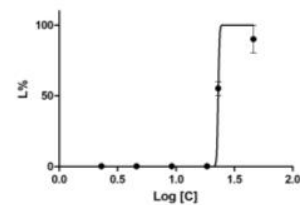

c

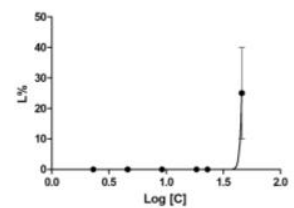

f

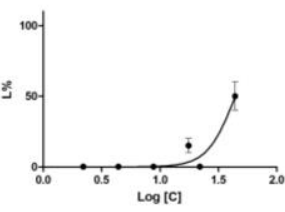

i

Figure 7.

Lethality curves on Daphnia magna at $72 \mathrm{~h}$ of exposure for tested compounds; $\mathrm{a}-\mathbf{3}, \mathrm{b}-\mathbf{4}, \mathrm{c}-\mathbf{6 b}, \mathrm{d}-\mathbf{6 c}, \mathrm{e}-\mathbf{7 a}, \mathrm{f}-\mathbf{7 b}, \mathrm{g}-\mathbf{7 c}, \mathrm{h}-$ phenylalanine, $\mathrm{i}-\mathbf{1}$

The $72 \mathrm{~h}$ decreasing order of toxicity of tested compounds was: $\mathbf{7 a}, \mathbf{6 c}, \mathbf{4}, \mathbf{7 b}, \mathbf{6 b}, \mathbf{3}, \mathbf{1}$ and $\mathbf{7 c}$. Phenylalanine induced a maximum of $20 \%$ lethality on daphnids at $72 \mathrm{~h}$. Values obtained experimentally differs greatly from those predicted with GUSAR software. Not only that at $48 \mathrm{~h}$ of exposure, $\mathrm{LC}_{50}$ couldn't be calculated for most compounds, but $\mathrm{LC}_{50}$ values obtained at $72 \mathrm{~h}$ are much lower than those predicted at $48 \mathrm{~h}$. Given the results obtained at $72 \mathrm{~h}$, the toxicity of compounds could be similar due to values obtained for $95 \% \mathrm{CI}$ which overlap. Compounds 7a and $\mathbf{1}$ can be considered most toxic on D. magna because they exhibited toxicity on $48 \mathrm{~h}$.

\section{Conclusions}

Eight new compounds from $N$-acyl- $\alpha$-amino acids, $N$-acyl- $\alpha$-amino acyl chlorides, 1,3-oxazol-5(4H)-ones, $\mathrm{N}$-acyl- $\alpha$-amino ketones and 1,3-oxazoles classes, bearing a 4-(4-chlorophenylsulfonyl)phenyl moiety, were synthesized and characterized. 2-Aryl-4-benzyl1,3-oxazol-5(4H)-one 4 was obtained by $N$-acylation of phenylalanine with acyl chloride 2 , followed by intramolecular cyclization of acyclic intermediate $\mathbf{3}$. $N$-Acyl- $\alpha$-amino acyl chloride $\mathbf{5}$ was also produced from compound 3. $N$-Acyl- $\alpha$-amino ketones $\mathbf{6}$ were synthesized by treatment of saturated 1,3-oxazol$5(4 H)$-one 4 with arenes in the presence of $\mathrm{AlCl}_{3}$. Also, the acylation of benzene with $\mathbf{5}$ was performed. By $\mathrm{POCl}_{3}$ and/or $\mathrm{H}_{2} \mathrm{SO}_{4}$-catalysed cyclodehydrations of acyclic precursors 6, the 2,5-diaryl-4-benzyl-1,3oxazoles 7 were obtained. Chemical structures of synthesized compounds were confirmed by elemental analysis and different spectral techniques.

New compounds $\mathbf{3}, \mathbf{4}, \mathbf{6 b}, \mathbf{c}, \mathbf{7 a - c}$ have been investigated for their toxicity and biological activity on Daphnia magna. All compounds exhibited cytotoxicity at 72 $\mathrm{h}$ of exposure, the effect being dependent on the exposure period. 1,3-Oxazole 7a showed the highest toxicity.

\section{Conflict of interest}

The authors declare no conflict of interest.

\section{References}

1. Zhang HZ, Zhao ZL, Zhou CH, Recent advance in oxazole-based medicinal chemistry. Eur J Med Chem., 2018; 144: 444-492.

2. Ansari A, Ali A, Asif M, Rauf MA, Owais M, Shamsuzzaman, Facile one-pot multicomponent synthesis and molecular docking studies of steroidal oxazole/thiazole derivatives with effective antimicrobial, antibiofilm and hemolytic properties. Steroids, 2018; 134: 22-36.

3. Abhale YK, Sasane AV, Chavan AP, Shekh SH, Deshmukh KK, Bhansali S, Nawale L, Sarkar D, Mhaske PC, Synthesis and antimycobacterial screening of new thiazolyl-oxazole derivatives. Eur J Med Chem., 2017; 132: 333-340.

4. Massaro M, Scoditti E, Pellegrino M, Carluccio MA, Calabriso N, Wabitsch M, Storelli C, Wright M, De Caterina R, Therapeutic potential of the dual peroxisome proliferator activated receptor (PPAR) $\alpha / \gamma$ agonist aleglitazar in attenuating TNF- $\alpha$-mediated inflammation and insulin resistance in human adipocytes. Pharmacol Res., 2016; 107: 125-136. 
FARMACIA, 2021, Vol. 69, 2

5. Semenyuta I, Kovalishyn V, Tanchuk V, Pilyo S, Zyabrev V, Blagodatnyy V, Trokhimenko O, Brovarets $\mathrm{V}$, Metelytsia L, 1,3-Oxazole derivatives as potential anticancer agents: Computer modeling and experimental study. Comput Biol Chem., 2016; 65: 8-15.

6. Tilvi S, Singh KS, Synthesis of oxazole, oxazoline and isoxazoline derived marine natural products: A Review. Curr Org Chem., 2016; 20(8): 898-929.

7. Swain SS, Paidesetty SK, Padhy RN, Antibacterial, antifungal and antimycobacterial compounds from cyanobacteria. Biomed Pharmacother., 2017; 90: 760-776.

8. La Regina G, Coluccia A, Naccarato V, Silvestri R, Towards modern anticancer agents that interact with tubulin. Eur J Pharm Sci., 2019; 131: 58-68.

9. Giddens AC, Boshoff HIM, Franzblau SG, Barry CE, Copp BR, Antimycobacterial natural products: synthesis and preliminary biological evaluation of the oxazole-containing alkaloid texaline. Tetrahedron Lett., 2005; 46(43): 7355-7357.

10. Apostol TV, Barbuceanu SF, Olaru OT, Draghici C, Saramet G, Socea B, Enache C, Socea LI, Synthesis, Characterization and Cytotoxicity Evaluation of New Compounds from Oxazol-5(4H)-ones and Oxazoles Class Containing 4-(4-Bromophenylsulfonyl)phenyl Moiety. Rev Chim., 2019; 70(4): 1099-1107.

11. Bala S, Saini M, Kamboj S, Methods for synthesis of Oxazolones: A Review. Int J ChemTech Res., 2011; 3(3): 1102-1118.

12. Pinto IL, West A, Debouck CM, Dilella AG, Gorniak JG, O'Donnell KC, O'Shannessy DJ, Patel A, Jarvest RL, Novel, selective mechanism-based inhibitors of the herpes proteases. Bioorg Med Chem Lett., 1996; 6(20): 2467-2472.

13. Apostol TV, Draghici C, Dinu M, Barbuceanu SF, Socea LI, Saramet I, Synthesis, Characterization and Biological Evaluation of New 5-aryl-4-methyl-2[para-(phenylsulfonyl)phenyl]oxazoles. Rev Chim., 2011; 62(2): 142-148.

14. Cazzola M, Calzetta L, Page C, Rogliani P, Matera MG, Thiol-Based Drugs in Pulmonary Medicine: Much More than Mucolytics. Trends Pharmacol Sci., 2019; 40(7): 452-463.

15. Wong D, Tsai PNW, Ip KY, Irwin MG, New antihypertensive medications and clinical implications. Best Pract Res Clin Anaesthesiol., 2018; 32(2): 223-235.

16. Abdelrady H, Hathout RM, Osman R, Saleem I, Mortada ND, Exploiting gelatin nanocarriers in the pulmonary delivery of methotrexate for lung cancer therapy. Eur J Pharm Sci., 2019; 133: 115-126.

17. Argyridis S, Folic acid in pregnancy. Obstet Gynaecol Reprod Med., 2019; 29(4): 118-120.

18. Sharma P, Singh S, Siddiqui TI, Singh VS, Kundu B, Prathipati P, Saxena AK, Dikshit DK, Rastogi L, Dixit C, Gupta MB, Patnaik GK, Dikshit M, $\alpha-$ Amino acid derivatives as proton pump inhibitors and potent anti-ulcer agents. Eur J Med Chem., 2007; 42(3): 386-393.

19. Jadhav AP, Sadaka FG, Angiotensin II in septic shock. Am J Emerg Med., 2019; 37(6): 1169-1174.

20. Ogwang S, Nguyen HT, Sherman M, Bajaksouzian S, Jacobs MR, Boom WH, Zhang GF, Nguyen L,
Bacterial Conversion of Folinic Acid Is Required for Antifolate Resistance. J Biol Chem., 2011; 286(17): 15377-15390.

21. Schaper A, Ebbecke M, Intox, detox, antidotes Evidence based diagnosis and treatment of acute intoxications. Eur J Intern Med., 2017; 45: 66-70.

22. Fear G, Komarnytsky S, Raskin I, Protease inhibitors and their peptidomimetic derivatives as potential drugs. Pharmacol Ther., 2007; 113(2): 354-368.

23. Semple G, Ashworth DM, Batt AR, Baxter AJ, Benzies DWM, Elliot LH, Evans DM, Franklin RJ, Hudson P, Jenkins PD, Pitt GR, Rooker DP, Yamamoto $\mathrm{S}$, Isomura Y, Peptidomimetic aminomethylene ketone inhibitors of interleukin-1 $\beta$-converting enzyme (ICE). Bioorg Med Chem Lett., 1998; 8(8): 959-964.

24. Deng H, Bannister TD, Jin L, Babine RE, Quinn J, Nagafuji P, Celatka CA, Lin J, Lazarova TI, Rynkiewicz MJ, Bibbins F, Pandey P, Gorga J, Meyers HV, AbdelMeguid SS, Strickler JE, Synthesis, SAR exploration, and X-ray crystal structures of factor XIa inhibitors containing an $\alpha$-ketothiazole arginine. Bioorg Med Chem Lett., 2006; 16(11): 3049-3054.

25. Bera S, Mondal D, Insights of synthetic analogues of anti-leprosy agents. Bioorg Med Chem., 2019; 27(13): 2689-2717.

26. Mishra M, Mishra VK, Kashaw V, Iyer AK, Kashaw SK, Comprehensive review on various strategies for antimalarial drug discovery. Eur J Med Chem., 2017; 125: $1300-1320$.

27. Noordeen SK, History of chemotherapy of leprosy. Clin. Dermatol., 2016; 34(1): 32-36.

28. Mady MF, Awad GEA, Jørgensen KB, Ultrasoundassisted synthesis of novel 1,2,3-triazoles coupled diaryl sulfone moieties by the $\mathrm{CuAAC}$ reaction, and biological evaluation of them as antioxidant and antimicrobial agents. Eur J Med Chem., 2014; 84: 433-443.

29. Wozel VEG, Innovative Use of Dapsone. Dermatol Clin., 2010; 28(3): 599-610.

30. Pezzella AT, Fang W, Surgical Aspects of Thoracic Tuberculosis: A Contemporary Review-Part 1. Curr Probl Surg., 2008; 45(10): 675-758.

31. Roșca EV, Apostol TV, Chifiriuc MC, Grădișteanu Pîrcălăbioru G, Drăghici C, Socea LI, Olaru OT, Nițulescu GM, Pahonțu EM, Hrubaru M, Bărbuceanu ŞF, In silico and experimental studies for the development of novel oxazol-5(4H)-ones with pharmacological potential. Farmacia, 2020; 68(3): 453-462.

32. Ahmad I, Shagufta, Sulfones: an important class of organic compounds with diverse biological activities. Int J Pharm Pharm Sci., 2015; 7(3): 19-27.

33. Apostol TV, Saramet I, Draghici C, Barbuceanu SF, Socea LI, Almajan GL, Synthesis and Characterization of New 5-Aryl-2-[para-(4-chlorophenylsulfonyl)phenyl]4-methyloxazoles. Rev Chim., 2011; 62(5): 486-492.

34. Mavrodin A, Oteleanu D, Stoenescu MV, Zotta V, Studies on the sulfone group. IV. New sulfonehydrazide derivatives. Pharm Zentralhalle Dtschl., 1956; 95(9): 353-361.

35. Schiketanz I, Draghici C, Saramet I, Balaban AT, Aminoketone, oxazole and thiazole synthesis. Part $15 .{ }^{1}$ 2-[4-(4-Halobenzenesulphonyl)-phenyl]-5-aryloxazoles. Arkivoc, 2002; (ii): 64-72. 